\title{
ESTABILIDAD DEL RENDIMIENTO DE GRANO EN CEBADA MALTERA EN EL BAJÍO, MÉXICO
}

\section{YIELD GRAIN STABILITY IN MALTING BARLEY IN EL BAJIO, MEXICO}

\author{
Juan Pérez-Ruiz ${ }^{*}$, Mauro Zamora-Díaz ${ }^{1}$, José Mejía-Contreras² ${ }^{2}$ Adrián Hernández-Livera² ${ }^{2}$ y \\ Salomón Solano-Hernández ${ }^{3}$
}

${ }^{1}$ Campo Experimental Valle de México, Instituto Nacional de Investigaciones Forestales, Agrícolas y Pecuarias (INIFAP), Carretera Los Reyes-Lechería, km 18.5, Coatlinchan, Texcoco, Estado de México, México. C.P. 56250.

2 Postgrado en Producción de Semillas, Colegio de Postgraduados, km. 36.5 Carretera México-Texcoco, Montecillo, Texcoco, Estado de México, México. C.P. 56230.

${ }^{3}$ Campo Experimental Bajío, INIFAP, Carretera Celaya-San Miguel de Allende S/N, km 6.5, Col. Roque, Celaya, Guanajuato, México, C.P. 38110.

* Autor para correspondencia E-mail: juan.perez@colpos.mx

\section{RESUMEN}

La interacción genotipo $x$ ambiente ( $\mathrm{G} \times \mathrm{A}$ ) está asociada al comportamiento diferencial que presentan los genotipos bajo diferentes condiciones de desarrollo y años de evaluación. El objetivo de este estudio fue evaluar la interacción genotipo $\mathrm{x}$ ambiente $\mathrm{y}$ estabilidad del rendimiento de grano de diez genotipos de cebada maltera mediante el modelo de efectos principales aditivos e interacción multiplicativa (AMMI). El estudio se realizó en El Bajío, México, que es la zona de mayor productividad y se ubica en la región Centro Occidente del país. Los genotipos se sembraron en cinco fechas de siembra y dos ciclos de cultivo: noviembre 15 y 30, diciembre 15 y 30 y enero 15, bajo condiciones de riego, en los ciclos otoño-invierno 2012-2013 y 2013-2014, generando diez ambientes de prueba. El modelo AMMI mostró diferencias significativas para ambientes, genotipos y para la interacción G x A, siendo congruente con lo obtenido en el ANOVA; estos resultados confirman la existencia de una fuerte variación de las condiciones ambientales en las diferentes fechas de siembra y años de prueba. Los genotipos Adabella y Esmeralda interaccionaron poco con el ambiente, pero su rendimiento fue inferior a la media general. El genotipo M-174 tuvo poca interacción con el ambiente y su rendimiento superó la media general, en tanto que los demás genotipos interactuaron más con el ambiente. Los genotipos seleccionados para condiciones de riego mostraron mayor potencial de rendimiento en las siembras correspondientes al 15 y 30 de noviembre y 15 de diciembre. Por otra parte, los genotipos para secano mostraron mejor respuesta a la variación ambiental, pero su rendimiento fue inferior a la media general.

Palabras clave: Hordeum vulgare L., interacción genotipo x ambiente, modelo AMMI, fechas de siembra.

\section{ABSTRACT}

Genotype $x$ environment interaction ( $\mathrm{G} \times \mathrm{A}$ ) is associated with the differential performance of genotypes under different development conditions and testing years. The aim of this study was to evaluate the genotype $x$ environment interaction and grain yield stability in ten malting barley genotypes by using the additive main effects and multiplicative interaction method (AMMI). 
The study was conducted in El Bajio (Mexico), which is located in the Central West region and corresponds to the country's most productive area. The genotypes were sown in five different sowing dates and two growing cycles: November 15 (FS1) and 30 (FS2), December 15 (FS3) and 30 (FS4) and January 15 (FS5), under irrigation in autumn-winter 2012-2013 and 2013-2014 cycles, generating ten test environments. The results obtained with the AMMI model showed significant differences for environments, genotypes and G x A interactions, which were consistent with those obtained in the ANOVA. This confirms that there is a strong variation in environmental conditions at different planting dates and years of testing. 'Esmeralda' and 'Adabella' interacted little with the environment, but their performance was lower than the general average. Genotype M-174 had little interaction with the environment and its yield was higher than the general average while the other genotypes presented larger interactions with the environment. The genotypes selected for irrigation conditions showed higher yield potential in the November 15 and 30 and December 15 planting dates. Moreover, the genotypes selected for conditions of dry land showed better response to environmental variation, but their performance was lower than the general average.

Key words: Hordeum vulgare L., genotype x environment interaction, AMMI model, planting dates.

\section{INTRODUCCIÓN}

En México, aproximadamente el $80 \%$ de la producción nacional de grano de cebada se produce en el ciclo primavera-verano en siembras de secano en los Valles Altos del país, y el resto de la producción de obtiene bajo condiciones de riego en el ciclo otoño-invierno en la región de El Bajío, que es la zona de mayor productividad del país, con rendimiento promedio de $6,3 \mathrm{t} \mathrm{ha}^{-1}$. En El Bajío se siembra una superficie superior a sesenta mil hectáreas. Esta región se ubica en el Centro Occidente del país y comprende parte de los estados de Querétaro, Guanajuato, Michoacán y Jalisco (SIAP, 2014).

En los programas de mejoramiento genético el rendimiento de grano es el principal objetivo de selección, destinado a incrementar el potencial de rendimiento y lograr su adaptación en ambientes sujetos a condiciones de estrés (Araus et al., 2008). El rendimiento de un cultivo es el resultado final de una compleja interrelación entre los procesos de crecimiento y desarrollo de los cultivos, fuertemente afectados por factores genéticos y ambientales, y principalmente por la interacción de ambos (Slafer, 2007).

La evaluación del rendimiento en entornos que van desde condiciones óptimas a condiciones severas de estrés permiten conocer la capacidad de adaptación de los genotipos a un amplio rango de condiciones ambientales (Araus et al., 2008). La variación del rendimiento se atribuye al potencial productivo del ambiente y al potencial genético en interacción con el primero (Hortelano et al., 2013). La interacción genotipo x ambiente se refiere al comportamiento diferencial de genotipos a través de condiciones ambientales variables, y es muy importante en el mejoramiento genético de los cultivos, debido a que está presente durante el proceso de selección y recomendación de genotipos (Rodríguez et al., 2011).

El termino estabilidad se utiliza para caracterizar a un genotipo que demuestra un rendimiento relativamente constante, a través de un amplio rango de condiciones ambientales (Eberhart y Russell, 1966). Actualmente existen diversos procedimientos univariados y multivariados para el estudio de la estabilidad. Entre los métodos univariados destaca el modelo de Eberhart y Russell (1966); sin embargo, se sugiere que los métodos multivariados son más adecuados para interpretar los efectos de la interacción genotipo $x$ ambiente, siendo el modelo de efectos principales aditivos e interacción multiplicativa (AMMI) uno de los métodos más empleados para el estudio de la estabilidad del rendimiento en cereales de grano pequeño como la cebada (Nurminiemi et al., 2002) y el trigo (Rodríguez et al., 2011; Negash et al., 2013), debido a que facilita la identificación visual del comportamiento de los genotipos y ambientes de evaluación mediante la gráfica biplot. Vargas y Crossa (2000) indican y ejemplifican la ventaja de la gráfica biplot para explicar la interacción genotipo $\mathrm{x}$ ambiente, cuando en el análisis de componentes principales un genotipo presenta un valor próximo a cero, la interacción es pequeña.

El modelo AMMI consiste en combinar las técnicas del análisis de varianza y el análisis de componentes principales en un solo modelo. El análisis de varianza permite estudiar los efectos principales de genotipos y ambientes, mientras que la interacción genotipo $x$ ambiente es tratada en forma multivariada mediante el análisis de componentes principales, realizando una reparametrización del modelo de regresión para mejorar la interpretación de la interacción genotipo $x$ ambiente. El objetivo del presente trabajo fue evaluar la interacción genotipo $\mathrm{x}$ ambiente $\mathrm{y}$ la estabilidad del rendimiento de diez genotipos 
de cebada maltera cultivados en la región de El Bajío, México, a través de cinco fechas de siembra y dos ciclos de cultivo.

\section{MATERIALES Y MÉTODOS}

Se evaluaron diez genotipos de cebada maltera (Hordeum vulgare L.) pertenecientes al Programa Nacional de Cebada, del Instituto Nacional de Investigaciones Forestales, Agrícolas y Pecuarias (INIFAP). Estos genotipos producen grano adecuado para la producción de malta, tienen buen potencial de rendimiento y presentan tolerancia a las principales enfermedades del cultivo; cinco de ellos son variedades comerciales y de disponibilidad comercial de semilla, y los otros cinco son líneas experimentales. Las variedades comerciales eran Alina, Armida, Esperanza, Adabella y Esmeralda; las primeras tres fueron liberadas para su cultivo en condiciones de riego en El Bajío y las dos restantes para condiciones de secano en los Valles Altos del país (Zamora et al., 2008; Solano et al., 2009; Zamora et al., 2010). Las líneas experimentales eran M-173, M-174, M-10542, M-176 y M-177; las tres primeras fueron seleccionadas para El Bajío y las dos restantes para los Valles Altos (Zamora, 2013. INIFAP, Programa Nacional de Cebada. Comunicación personal).

La evaluación del material experimental se realizó en el Campo Experimental Bajío (CEBAJ), perteneciente al INIFAP. El CEBAJ se localiza en Celaya, Guanajuato, México, a $20^{\circ} 32^{\prime} \mathrm{N}, 100^{\circ} 48^{\prime}$ W y $1752 \mathrm{msnm}$, la precipitación y temperatura media anual es de $578 \mathrm{~mm}$ y $19,8^{\circ} \mathrm{C}$, respectivamente.

Se establecieron cinco fechas de siembra: noviembre 15 y 30, diciembre 15 y 30, y enero 15 , en los ciclos otoño-invierno de 2012-2013 y 20132014. Cada fecha de siembra representó un ambiente de prueba, generándose diez ambientes de prueba, correspondiendo los ambientes del 1 al 5 al ciclo de 2012-2013 (A1, A2, A3, A4 y A5, respectivamente), y del 6 al 10 al ciclo de 2013-2014 (A6, A7, A8, A9 y A10, respectivamente). Cada unidad experimental consistió de cuatro surcos de $3,0 \mathrm{~m}$ de longitud y separación de $0,3 \mathrm{~m}$, la densidad de siembra fue de $100 \mathrm{~kg} \mathrm{ha}^{-1}$, con excepción de la variedad Esperanza que fue de $120 \mathrm{~kg} \mathrm{ha}^{-1}$, de acuerdo con la recomendación técnica debido a su capacidad baja de amacollamiento. Los riegos fueron aplicados al momento de la siembra, a los 45, 70 y 90 días después del primer riego.

El manejo agronómico empleado fue el recomendado por INIFAP para la región de El Bajío (Zamora et al., 2010). Se utilizó un diseño experimental de bloques completos al azar con tres repeticiones, bajo un arreglo factorial de tratamientos con tres factores de estudio; fechas de siembra, año de cultivo y genotipos. Cuando los genotipos alcanzaron la madurez fisiológica, se procedió a trillar los dos surcos centrales de cada unidad experimental con una minicombinada para parcelas experimentales.

\section{Variables evaluadas}

Contenido de humedad del grano. Se determinó mediante el uso de tres repeticiones de 120 gramos por unidad experimental, mediante un determinador de humedad digital (Burrows, Modelo DMC750, Chicago, EE.UU.).

Rendimiento de grano. Después de obtener el contenido de humedad del grano, se procedió a pesar el grano cosechado y obtener una corrección del contenido de humedad del grano para ajustar el rendimiento a $13 \%$ de humedad, y posteriormente se hizo la transformación a $t \mathrm{ha}^{-1}$.

\section{Análisis estadístico}

Se empleó el programa estadístico SAS (2009). Se realizó un análisis de varianza (ANVA) y se empleó el modelo de efectos principales aditivos e interacción multiplicativa (AMMI), el cual considera el análisis de varianza para efectos de genotipo y ambiente, y un análisis de componentes principales para la interacción genotipo $\mathrm{x}$ ambiente. El modelo matemático fue el siguiente:

$$
Y_{i j}=\mu+g_{i}+e_{j}+\sum_{k=1}^{n} \lambda_{k} \alpha_{i k} \gamma_{j k}+E_{i j}
$$

dónde: $Y_{i j}=$ rendimiento del i-ésimo genotipo en el j-ésimo ambiente; $\mu=$ media general; $g_{i}=$ efecto del i-ésimo genotipo; $e_{j}=$ efecto del j-ésimo ambiente; $\lambda_{k}=$ raíz cuadrada del vector característico del k-ésimo eje del ACP; $\alpha_{\text {ik }}=$ calificación del ACP para el k-ésimo eje del i ésimo genotipo; $Y_{\mathrm{j} k}=\mathrm{ca}$ lificación del ACP para el k-ésimo eje del j-ésimo ambiente; $\mathrm{E}_{\mathrm{ij}}=$ valor del error (Vargas y Crossa, 2000).

\section{RESULTADOS Y DISCUSIÓN}

Para rendimiento de grano se encontraron diferencias estadísticas para ambientes (A) en el análisis de varianza (ANVA). El efecto del ambiente contribuyó con $63,4 \%$ de la variación total de la suma de cuadrados (Tabla 1), debido a que la expresión del rendimiento se atribuye principalmente a los efectos ambientales durante el ciclo biológico del cultivo (Alam et al., 2007). Para genotipos $(G)$ hubo significancia, su efecto contribuyó con $22,7 \%$ de la suma de cuadrados (Tabla 1), manifestando que el potencial de rendimiento está influenciado por factores genéticos 
Tabla 1. Análisis de varianza combinado y significancia estadística para diez genotipos de cebada maltera evaluados en diez ambientes en la región de El Bajío, México.

Table 1. Combined analysis of variance and statistical significance of ten malting barley genotypes evaluated in ten environments in El Bajio region, Mexico.

\begin{tabular}{lcccc}
\hline FV & GL & SC & CM & SC (\%) \\
\hline Ambiente (A) & 9 & 679,1 & $75,45^{*}$ & 63,4 \\
Genotipo (G) & 9 & 243,2 & $27,02^{*}$ & 22,7 \\
A x G & 81 & 122,0 & $1,50^{*}$ & 11,4 \\
Error & 50 & 27,3 & 0,55 & 2,5 \\
Total corregido & 149 & 1071,5 & & \\
CV & 11,3 & & & \\
\hline
\end{tabular}

$\mathrm{FV}=$ factor de variación; $\mathrm{GL}=$ grados de libertad; $\mathrm{SC}=$ suma de cuadrados; $\mathrm{CM}=$ cuadrados medios; $\mathrm{CV}=$ coeficiente de variación.

* Significativo $(0,05)$

del cultivar y que se expresa cuando las condiciones ambientales son favorables. También hubo diferencias significativas para la interacción $\mathrm{G} \times \mathrm{A}$; el efecto de esta interacción contribuyó con $11,4 \%$ de la suma de cuadrados (Tabla 1), esto se debe a que la evaluación de genotipos bajo diversas condiciones ambientales, muestra una amplia variabilidad entre genotipos a través de los ambientes de prueba (Saad et al., 2013).

En el modelo AMMI se encontraron diferencias significativas para ambientes (A); su efecto contribuyó con $65,7 \%$ de la variación total de la suma de cuadrados (Tabla 2), siendo similar a la variación encontrada en el ANVA. Estos resultados confirman la existencia de fuerte variación de las condiciones ambientales entre fechas de siembra y ciclos de cultivo, influyendo considerablemente en la variación del rendimiento de grano.

Los resultados de este estudio concuerdan con los obtenidos por Negash et al. (2013) quienes evaluando la estabilidad del rendimiento de grano en trigo, encontraron que el efecto que contribuyó más a la varianza total fue el correspondiente al ambiente. El rendimiento en cereales de grano pequeño suele ser mayor en ambientes frescos que en condiciones cálidas, lo que coincidió con las tres primeras fechas de siembra de este estudio; y más cálidas en las dos últimas fechas de siembra, en ambos ciclos de cultivo. Para genotipos $(\mathrm{G})$ también hubo diferencias significativas, el efecto de genotipos contribuyó con 18,1 $\%$ de la variación total de la suma de cuadrados (Tabla 2) y fue relativamente inferior a lo encontrado en el ANVA.

Los genotipos generados para condiciones de riego mostraron mejor comportamiento en rendimiento de grano en comparación a los genotipos desarrollados para condiciones de secano (Tabla 3), debido a que el potencial de rendimiento depende del genotipo y de la región para la cual fue desarrollado (Soleymani y Shahrajabian, 2012).

La interacción $\mathrm{G} \times \mathrm{A}$ también fue significativa, el efecto de esta interacción contribuyó con $8,3 \%$ de la variación total de la suma de cuadrados (Tabla 2), siendo similar a lo encontrado en el ANVA. Estos resultados muestran diferencias en rendimiento entre genotipos a través de los diferentes ambientes de prueba y concuerdan con lo reportado por Bahrami et al. (2008), atribuido a que el rendimiento es fuertemente afectado por factores genéticos, ambientales, y principalmente por su interacción (Slafer, 2007). Los resultados obtenidos muestran que dentro de la variación total, los efectos de variación debido a la interacción $\mathrm{G}$ x A estuvieron presentes, poniendo de manifiesto la dificultad de lograr un rendimiento estable y poder predecir el comportamiento de los genotipos al ser evaluados (Solano et al., 1998).

Uno de los principales problemas en la evaluación de genotipos en un año y lugar específico, es que el efecto del lugar puede ser variable tanto en fechas de siembra como en años de evaluación (Bahrami et al., 2008). En este estudio el efecto significativo de la interacción $\mathrm{G} \times \mathrm{A}$ fue similar a lo encontrado en evaluaciones realizadas en cebada (Nurminiemi et al., 2002), trigo (Rodríguez et al., 2011; Castillo et al., 2012), sorgo (Williams et al., 2010) y maíz (San Vicente et al., 2005).

El modelo AMMI mostró que los cuadrados medios de los tres primeros componentes principales fueron significativos. El primer componente principal (CP1) explicó el 39,1\% de la suma de cuadrados de la interacción $\mathrm{G} \times \mathrm{A}$, mientras que el segundo componente principal (CP2) explicó el 33,2\% y el tercer componente principal (CP3) explicó el 15,4\% (Tabla 2). La magnitud del valor del CP1 fue suficiente para proveer una clara explicación de los resultados y visualizar la adaptación de los genotipos de cebada en los distintos ambientes de prueba (Fig.1). En este sentido, So- 
Tabla 2. Análisis de varianza AMMI y significancia estadística de diez genotipos de cebada maltera evaluados en diez ambientes en la región de El Bajío, México.

Table 2. AMMI analysis of variance and statistical significance of ten malting barley genotypes evaluated in ten environments in El Bajío region, Mexico.

\begin{tabular}{lrrrr}
\hline FV & GL & SC & CM & SC (\%) \\
\hline Ambiente (A) & 9 & 1429,7 & $158,85^{*}$ & 65,7 \\
Genotipo (G) & 9 & 393,4 & $43,70 *$ & 18,1 \\
G x A & 81 & 181,1 & $2,23 *$ & 8,3 \\
CP1 & 17 & 70,9 & $4,16^{*}$ & 39,1 \\
CP2 & 15 & 60,1 & $4,00^{*}$ & 33,2 \\
CP3 & 13 & 28,0 & $2,15^{*}$ & 15,4 \\
Error & 198 & 171,8 & 0,86 & 7,9 \\
Total corregido & 299 & 2176,0 & & \\
CV & 14,2 & & & \\
\hline
\end{tabular}

$\mathrm{FV}=$ factor de variación; $\mathrm{GL}=$ grados de libertad; $\mathrm{SC}=$ suma de cuadrados; $\mathrm{CM}=$ cuadrados medios; $\mathrm{CV}=$ coeficiente de variación; $\mathrm{CP} 1=$ componente principal $1 ; \mathrm{CP} 2=$ componente principal $2 ; \mathrm{CP} 3=$ componente principal 3 .

* Significativo $(0,05)$

Tabla 3. Rendimiento de grano y contribución de los dos primeros componentes principales del modelo AMMI.

Table 3. Grain yield and contribution of the two first principal components of the AMMI model.

\begin{tabular}{|c|c|c|c|c|c|}
\hline Factores & & & $\operatorname{REN}\left(t h^{-1}\right)$ & CP1 & $\mathrm{CP} 2$ \\
\hline \multirow{10}{*}{ Genotipo } & Adabella & & 5,0851 & 0,1676 & $-0,1259$ \\
\hline & Alina & & 8,2233 & $-0,6437$ & 0,4654 \\
\hline & Armida & & 7,9716 & $-0,7930$ & $-0,7311$ \\
\hline & Esmeralda & & 5,8592 & 0,1593 & $-0,0014$ \\
\hline & Esperanza & & 7,1746 & $-0,6883$ & 0,6801 \\
\hline & M-173 & & 5,7540 & 0,5851 & $-1,4704$ \\
\hline & M-174 & & 6,8087 & 0,0460 & 0,0063 \\
\hline & M-176 & & 4,8033 & 1,5126 & 0,3932 \\
\hline & M-177 & & 6,2020 & 0,3734 & 0,9503 \\
\hline & M-10542 & & 7,7364 & $-0,7191$ & $-0,1664$ \\
\hline \multirow[t]{5}{*}{ Ciclo 2012-2013 } & Nov-15 & A1 & 6,8503 & 0,2799 & 0,4201 \\
\hline & Nov-30 & A2 & 7,7273 & $-0,3509$ & $-0,3597$ \\
\hline & Dic-15 & A3 & 4,8449 & $-1,0013$ & $-0,3015$ \\
\hline & Dic-30 & A4 & 4,8755 & $-0,1753$ & $-0,3879$ \\
\hline & Ene-15 & A5 & 4,6146 & 0,6235 & $-0,0844$ \\
\hline \multirow[t]{5}{*}{ Ciclo 2013-2014 } & Nov-15 & A6 & 9,8949 & $-0,3492$ & 1,5910 \\
\hline & Nov-30 & A7 & 9,8266 & $-0,6125$ & 0,4847 \\
\hline & Dic-15 & A8 & 7,4789 & $-0,3527$ & $-0,9211$ \\
\hline & Dic-30 & A9 & 6,6862 & 0,3630 & $-0,5455$ \\
\hline & Ene-15 & A10 & 2,8193 & 1,5756 & 0,1044 \\
\hline Media general & & & 6,5618 & & \\
\hline
\end{tabular}

REN = Promedio del rendimiento de grano; $\mathrm{A}=$ ambiente; $\mathrm{CP} 1$ = componente principal $1 ; \mathrm{CP} 2$ = componente principal 2. REN = average grain yield; $\mathrm{A}=$ environment; $\mathrm{CP} 1$ = principal component $1 ; \mathrm{CP} 2$ = principal component 2. 
lano et al. (1998) señalaron que el CP1 permite determinar el efecto de la interacción al predecir el comportamiento de un genotipo en un ambiente, y frecuentemente describe la mayor proporción de la interacción, por lo tanto, es el componente principal que mejor discrimina entre genotipos. Resultados similares a este estudio fueron obtenidos por Negash et al. (2013) quienes estudiando la interacción $\mathrm{G} x \mathrm{~A}$ en el rendimiento de trigo, $\mathrm{y}$ analizando de forma separada dos años de prueba mediante el modelo AMMI,

Las variedades Adabella y Esmeralda tuvieron valores bajos en el CP1, manifestando que interaccionaron poco con el ambiente, sin embargo, su rendimiento fue inferior a la media general $(6,5$ $t$ ha $\left.{ }^{-1}\right)$. El genotipo M-174 mostró un valor bajo en el CP1 y su rendimiento fue mayor a la media general, mostrando un comportamiento estable. Los genotipos M-173 y M-177 tuvieron valores relativamente bajos en el $\mathrm{CP} 1$, pero su rendimiento fue inferior a la media general; mientras que los genotipos Alina, Armida, Esperanza y M-10542 tuvieron rendimientos superiores a la media general, pero presentaron mayor interacción con el ambiente, debido a que mostraron valores altos en el CP1. El genotipo M-176 mostró un rendimiento inferior a la media general y fue el genotipo con mayor interacción con el ambiente (Fig. 1).

Rodríguez et al. (2011) mencionaron que los genotipos más estables se encuentran más cerca- nos al origen del CP1 y al alejarse de este muestran mayor interacción con el ambiente. Los resultados obtenidos concuerdan con San Vicente et al. (2005) quienes evaluando la estabilidad del rendimiento de grano en maíz encontraron que los genotipos con mayor estabilidad presentan rendimientos inferiores a la media general.

El ambiente A1 presentó un valor bajo en el $\mathrm{CP} 1$ y mostró rendimientos superiores a la media general $\left(6,5 \mathrm{t} \mathrm{ha}^{-1}\right)$, mientras que el ambiente A4 también tuvo valores bajos en el CP1, pero los rendimientos fueron inferiores a la media general; en ambos ambientes los genotipos se mostraron estables (Fig. 1). En el ambiente A9 hubo un rendimiento ligeramente superior a la media general, mientras que en los ambientes $\mathrm{A} 2, \mathrm{~A} 8$, A7 y A6 se presentaron rendimientos superiores a la media general, pero en todos los casos hubo valores relativamente altos en el CP1. Además, en los ambientes A3, A5 y A10 hubo rendimientos inferiores a la media general, siendo los dos últimos donde los genotipos evaluados mostraron mayor interacción (Fig. 1). Por otra parte, habría que considerar que las diferencias en rendimiento en los ambientes A1, A2 y A3 (ciclo 2012-2013), en comparación a los ambientes A6, A7 y A8 (ciclo 2013-2014) se debió a la presencia de heladas atípicas, las cuales se presentaron cuándo los genotipos se encontraban en la etapa de llenado de grano (A1 y A2) y espigamiento (A3), habien-

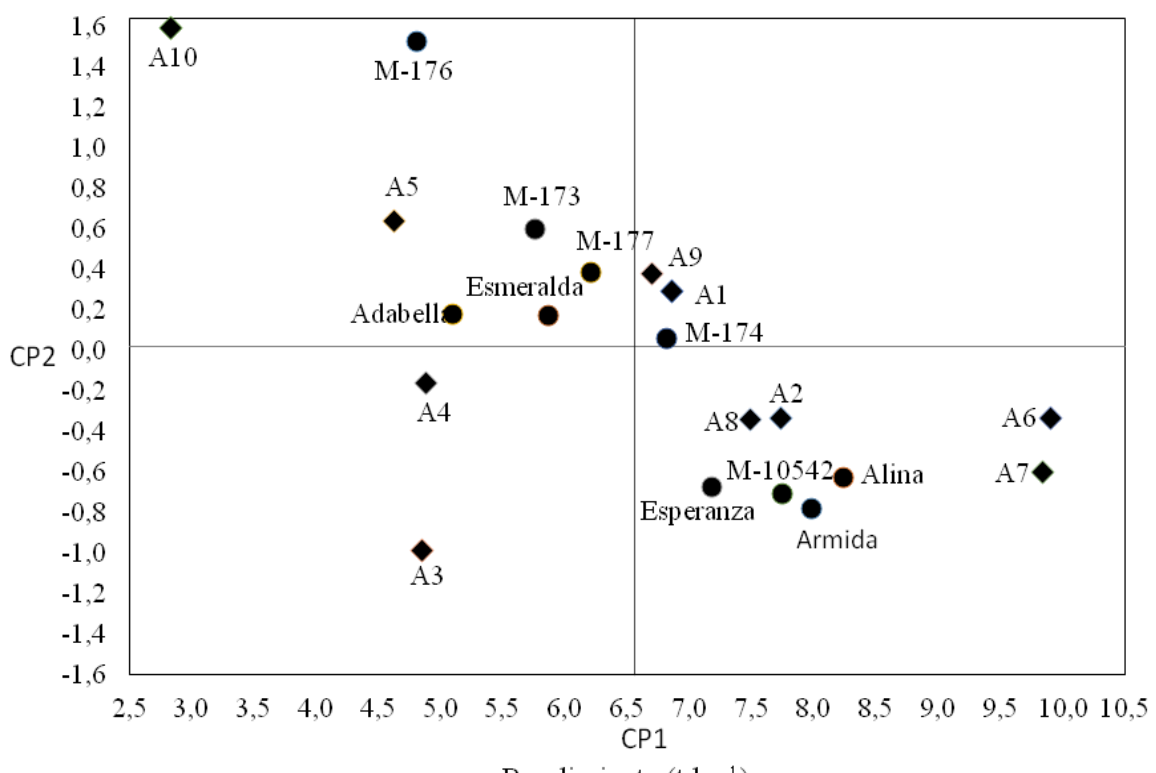

Rendimiento (t ha-1)

Fig. 1. Gráfica biplot del rendimiento de grano de diez genotipos de cebada maltera evaluados en diez ambientes en la región de El Bajío, México.

Fig. 1. Biplot graphical representation of the grain yield of ten malting barley genotypes evaluated in ten environments in El Bajío region, Mexico. 
do una merma de grano en todos los genotipos, observándose mayor daño en el ambiente A3. Nurminiemi et al. (2002) mencionaron que la variación de rendimiento entre ambientes puede atribuirse a factores como la ubicación geográfica de la localidad de prueba, fecha de siembra, aumento o descenso de temperatura, y susceptibilidad a enfermedades.

La variación en el rendimiento de grano de cebada se atribuyó a las condiciones ambientales particulares que predominaron en cada ambiente de prueba. Los genotipos Alina, Armida, Esperanza, M-174 y M-10542 seleccionados para condiciones de riego, expresaron mejor rendimiento de grano en los ambientes A1, A2, A6, A7 y A8 (correspondientes a siembras del 15 y 30 de noviembre de ambos ciclos de cultivo, y 15 de diciembre del ciclo 2013-2014, posiblemente porque las condiciones de estrés fueron menores en comparación a los ambientes A4, A5, A9 y A10 (siembras del 30 de diciembre y 15 de enero de ambos ciclos). En las siembras establecidas a inicios del invierno, el aumento de temperatura y baja humedad relativa coincidió con la etapa del llenado de grano, favoreciendo la pérdida de humedad del suelo a través de la evaporación, además del aumento de la transpiración del cultivo, lo cual demanda mayor número de riegos para evitar pérdidas en el rendimiento.

El genotipo M-173 tuvo un comportamiento similar a los genotipos Adabella, Esmeralda y M-177 seleccionados para condiciones de secano, los cuales a pesar de tener un rendimiento de grano inferior a la media general, mostraron mejor respuesta a la variación ambiental. Las variaciones observadas en el rendimiento de grano pudieran deberse a la interacción genotipo $\mathrm{x}$ ambiente. Saad et al. (2013) mencionan que los genotipos de cebada difieren en rendimiento cuando se evalúan bajo diferentes condiciones ambientales, en respuesta al efecto de la interacción genotipo $x$ ambiente.

\section{CONCLUSIONES}

El modelo AMMI mostró diferencias significativas para ambientes, genotipos e interacción G x A, siendo similar a lo obtenido en el ANVA; estos resultados confirman la existencia de fuerte variación de las condiciones ambientales entre fechas de siembra y años de prueba. El genotipo M-174 mostró poca interacción con el ambiente y su rendimiento de grano fue mayor a la media general. Los genotipos Alina, Armida y M-10542 generados para condiciones de riego mostraron mayor potencial del rendimiento pero fueron más inestables. Los genotipos Adabella, Esmeralda y M-177 generados para condiciones de secano mostraron mejor respuesta a la variación ambiental, pero su rendimiento fue inferior a la media general.

\section{LITERATURA CITADA}

Alam, M.Z., S.A. Haider, and N.K. Paul. 2007. Yield and yield components of barley (Hordeum vulgare L.) in relation to sowing times. Journal of Biological Sciences 15:139-145.

Araus, J.L, G.A. Slafer, C. Royo, and M.D. Serret. 2008. Breeding for yield potential and stress adaptation in cereals. Critical Reviews in Plant Sciences 27(6):377-412.

Bahrami, S., M.R. Bihamta, M. Salari, M. Soluki, A. Ghanbari, A.A. Vahabi Sadehi, and A. Kazemipour. 2008. Yield stability analysis in hulless barley (Hordeum vulgare L.). Asian Journal of Plant Sciences 7(6):589-593.

Castillo, D., I. Matus, A. del Pozo, R. Madariaga, and M. Mellado. 2012. Adaptability and genotype $\mathrm{x}$ environment interaction of spring wheat cultivars in Chile using Regression Analysis, AMMI, and SREG. Chilean Journal of Agricultural Research 72(2):167-174.

Eberhart, S., and W.A. Russell. 1966. Stability parameters for comparing varieties. Crop Science 6:36-40.

Hortelano, R., H.E. Villaseñor, E. Martínez, M.F. Rodríguez, E. Espitia, y L.A. Mariscal. 2013. Estabilidad de variedades de trigo recomendadas para siembras de temporal en los Valles Altos de la Mesa Central. Revista Mexicana de Ciencias Agrícolas 4(5):713-725.

Negash, A.W., H. Mwambi, T. Zewotir, and G. Taye. 2013. Additive main effects and multiplicative interactions model (AMMI) and genotype main effect and genotype by environment interaction (GGE) biplot analysis of multi-environmental wheat variety trials. African Journal of Agricultural Research 8(12):1033-1040.

Nurminiemi, M., S. Madsen, O.A. Rognli, A. Bjornstad, and R. Ortiz. 2002. Analysis of the genotype-by-environment interaction of spring barley tested in the Nordic Region of Europe: Relationships among stability statistics for grain yield. Euphytica 127:123-132.

Rodríguez, R.E., J.F. Ponce, E.O. Rueda, L. Avendaño, J.J. Paz, J. Santillano, y M. Cruz. 2011. Interacción genotipo-ambiente para la estabilidad de rendimiento en trigo en la región de Mexicali, B.C., México. Tropical and Subtropical Agroecosystems 14:543- 558.

Saad, F.A, A.A. Abd El-Mohsen, and I.H. Al-Soudan. 2013. Parametric statistical methods for evaluating barley genotypes in multi-environment trials. Scientia Agriculturae 1(2):30-39. 
San Vicente F.M., C. Marín R., y D. Díaz. 2005. Estabilidad del rendimiento y potencial agronómico de híbridos de maíz de alta calidad de proteína (QPM) en Venezuela. Agronomía Tropical 55(3):397-410.

SAS. 2009. Statistical Analysis System. Release 9.1 for Windows. SAS Institute Inc., Cary, North Carolina, USA.

SIAP. 2014. Secretaria de Agricultura, Ganadería, Desarrollo Rural, Pesca y Alimentación (SAGARPA). Servicio de Información Agroalimentaria y Pesquera (SIAP). Disponible en http://www.siap. gob.mx (Consultado 09 diciembre 2015).

Slafer, G.A. 2007. Physiology of determination of major wheat yield components. p. 557-565. In H.T. Buck, J.E. Nisi and N. Salomón (eds.). Wheat Production in Stressed Environments. Springer, Dordrecht, Netherlands.

Solano, S.J., B.P. Barriga, H.A. Krarup, y S.H. Figueroa. 1998. Estabilidad temporal del rendimiento de genotipos mutantes de trigo, mediante el modelo de interacción multiplicativa y efectos principales aditivos (AMMI: Additive main effect and multiplicative interaction model). Agro Sur 26(2):19-32.

Solano, S., M. Zamora, F.P. Gámez, J.J. García, R. Sánchez, J. Ireta, et al. 2009. Alina, nueva variedad de cebada maltera para riego en El Bajío. Agricultura Técnica en México 35(4):467469.
Soleymani, A., and M.H. Shahrajabian. 2012. Changes in seed yield and yield components of elite barley cultivars under different plant populations and sowing dates. Journal of Food, Agriculture and Environment 10(1):596-598.

Vargas, M., y J. Crossa. 2000. El análisis AMMI y la gráfica del biplot en SAS. 42 p. Unidad de Biometría y Estadística. CIMMYT, México.

Williams, H., V. Pecina, F. Zavala, N. Montes, A. J. Gámez, G. Arcos, et al. 2010. Modelo de Finlay y Wilkinson vs. el Modelo AMMI para analizar la interacción genotipo-ambiente en sorgo. Revista Fitotecnia Mexicana 33(2):117123.

Zamora, M., S. Solano, R. Gómez, I. Rojas, J. Ireta, R. Garza, y C. Ortiz. 2008. Adabella: variedad de cebada maltera para valles altos de la mesa central de México. Agricultura Técnica en México 34(4):491-493.

Zamora, M., S. Solano, R. Garza, J. Islas, R. Huerta, y M. López. 2010. Armida, nueva variedad de cebada maltera para riego en El Bajío. Revista Mexicana de Ciencias Agrícolas 1(5):723-726. 